\title{
Importância do diagnóstico precoce em crianças com Transtorno do Déficit de Atenção com Hiperatividade: revisão narrativa
}

\author{
Importance of early diagnosis in children with Attention Deficit Hyperactivity Disorder: \\ narrative review
}

\author{
Importancia del diagnóstico precoz en niños con el Trastorno por Déficit de Atención e \\ Hiperactividad: revisión narrativa
}

Emanuele Janoca Franca ${ }^{1 *}$, Rosilda Brunele da Silva Alves², Lívia Pêpe Leão da Rocha ${ }^{3}$, Beatriz Will Braga ${ }^{4}$, Emanuel Sampaio Borba Lana ${ }^{3}$, Ana Larissa Nunes Colares ${ }^{5}$, Ana Beatriz Ramos Lopes $^{3}$, Silvio Matheus Azevedo Rocha ${ }^{6}$, Maria Eduarda Costa Naves ${ }^{7}$, Gustavo Fonseca Genelhu Soares $^{2}$.

\section{RESUMO}

Objetivo: Discutir e revisar a importância do diagnóstico precoce do transtorno de Déficit de Atenção e Hiperatividade (TDAH) em crianças e seu tratamento adequado. Revisão bibliográfica: Os danos causados pelo transtorno incluem baixo desenvolvimento na competência escolar, relacionamentos conflituosos, problemas emocionais e de autoestima e comorbidades associadas como ansiedade e depressão. $O$ diagnóstico depende da utilização de escalas e entrevistas não apenas com o paciente, mas também com familiares e professores, conforme a idade (4 anos a 18 anos) segundo o Manual Diagnóstico e Estatístico de Transtornos Mentais (DSM-5) da Associação Psiquiátrica Americana e a Classificação de Transtornos Mentais e de Comportamento (CID-10). Considerações finais: Graças aos avanços e pesquisas, há um amplo arsenal terapêutico preenchido por derivados de metilfenidato ou de anfetaminas associados à terapia comportamental que é essencial para corroborar a diminuição da administração farmacológica nas crianças e consequentemente diminuindo os efeitos adversos de altas doses da medicação.

Palavras-chave: Transtorno de déficit de atenção e hiperatividade (TDAH), Crianças, Qualidade de vida da criança, Deficiências da aprendizagem.

\begin{abstract}
Objective: To discuss and review the importance of early diagnosis of Attention Deficit Hyperactivity Disorder $(A D H D)$ in children and its proper treatment. Bibliographic review: The damage caused by the disorder includes poor development in school competence, conflicting referrals, emotional and self-esteem problems and comorbidities associated with anxiety and depression. Diagnosis depends on the use of scales and choice not only with the patient, but also with family members and teachers, according to age (4 years to 18 years) according to the Diagnostic and Statistical Manual of Mental Disorders (DSM-5) of the American Psychiatric Association and the Classification of Mental and Behavioral Disorders (CID-10). Final considerations: Thanks to advances and research, there is a wide therapeutic arsenal filled with derivatives of methylphenidate or amphetamines associated with behavioral therapy, which is essential to support the decrease in drug administration in children and, consequently, reducing the adverse effects of high doses of medication.
\end{abstract}

Key words: Attention deficit hyperactivity disorder (ADHD), Children, Child's quality of life, Learning disabilities.

\footnotetext{
${ }^{1}$ Centro Universitário Aparício Carvalho (UNIFIMCA), Porto Velho - RO.

*E-mail: emanuelejanoca@gmail.com

${ }^{2}$ Centro Universitário de Caratinga (UNEC), Caratinga - MG.

${ }^{3}$ Instituto Metropolitano de Ensino Superior (UNIVAÇO), Ipatinga - MG.

${ }^{4}$ Faculdade de Ciências Biomédicas de Cacoal (UNIFACIMED), Cocal - RO.

${ }^{5}$ Universidade Nilton Lins (UNL), Manaus - AM.

6 Universidade Tiradentes, Aracajú - SE.

${ }^{7}$ Instituto Master de Ensino Presidente Antônio Carlos (IMEPAC), Araguari - MG.
} 


\section{RESUMEN}

Objetivo: Discutir y revisar la importancia del diagnóstico precoz del Trastorno por Déficit de Atención con Hiperactividad (TDAH) en niños y su adecuado tratamiento. Revisión bibliográfica: El daño causado por el trastorno incluye un desarrollo deficiente en la competencia escolar, derivaciones conflictivas, problemas emocionales y de autoestima y comorbilidades asociadas con la ansiedad y la depresión. El diagnóstico depende del uso de escalas y elección no solo con el paciente, sino también con familiares y profesores, según la edad (4 años a 18 años) según el Manual Diagnóstico y Estadístico de Trastornos Mentales (DSM5) de la Asociación Americana de Psiquiatría y Clasificación de Trastornos Mentales y del Comportamiento (CID-10). Consideraciones finales: Gracias a los avances y la investigación, existe un amplio arsenal terapéutico repleto de derivados del metilfenidato o anfetaminas asociados a la terapia conductual, que es fundamental para apoyar la disminución de la administración de medicamentos en los niños y, en consecuencia, reducir los efectos adversos de las dosis elevadas. de medicación.

Palabras clave: Trastorno por déficit de atención e hiperactividad (TDAH), Niños, Calidad de vida infantil, Dificultades de aprendizaje.

\section{INTRODUÇÃO}

O Manual Diagnóstico e Estatístico de Transtornos Mentais (DSM-5) (2014), estabelece o Transtorno do Déficit de Atenção com Hiperatividade (TDAH) como um incessante padrão de desatenção, hiperatividade e impulsividade que afeta o funcionamento do desenvolvimento neurocomportamental podendo gerar distúrbios motores, perceptivos e cognitivos (DSM-5, 2014). É consequência de um desarranjo genético que cursa com uma diminuição do aporte de neurotransmissores estimulantes no cérebro (SIGNOR RCF e SANTANA APO, 2020).

Esse transtorno de comportamento neurológico é um dos mais frequentes em crianças e tem repercussão negativa no cotidiano. Há vários tipos de comorbidades que podem ser identificadas juntamente ao TDAH, como: problemas de socialização, problemas familiares, problemas emocionais e de autoestima, dificuldades em habilidades cognitivas e de linguagem e problemas de conduta (EFFGEM V, et al., 2017).

Estudos epidemiológicos ressaltam que a prevalência mundial estimada do TDAH em crianças e adolescentes é em torno de 5,29\%. Conforme alguns pesquisadores essa prevalência aumentou nos últimos anos em variados países, juntamente com a prescrição de medicamentos utilizados em seu tratamento (CARVALHO DMS, et al., 2017).

O diagnóstico precoce do TDAH se faz necessário para que os danos causados pelo transtorno sejam evitados. Crianças portadoras do transtorno apresentam uma maior dificuldade de aprendizado e desenvolvimento nos âmbitos social e familiar. Além disso, há um maior risco de aparecimento de comorbidades como transtornos depressivos ou de personalidade. Os critérios diagnósticos envolvem sintomas apresentados, idade de início dos sintomas, configurações desses sintomas (desatenção e hiperatividade ou desatenção ou hiperatividade), duração dos sintomas, comprometimento de funcionalidade e subtipos. Além disso, uma avaliação detalhada em busca de informações sobre história da gravidez, comportamento na escola e no âmbito familiar devem ser realizados para uma maior segurança na definição do diagnóstico (BANASCHEWKI T, et al., 2017; FELDMAN HM, et al., 2014).

A utilização de drogas farmacológicas e terapia comportamental são úteis no tratamento das crianças portadoras de TDAH. No primeiro grupo, tem como arsenal terapêutico os medicamentos estimulantes derivados de metilfenidato ou derivados de anfetaminas. Essas medicações podem ajudar na melhora da concentração e diminuição da hiperatividade, porém trazem consigo efeitos colaterais como ansiedade, perda de apetite e desregulação do sono. A perda de apetite, em especial, é um efeito colateral que causa preocupação, pois estamos falando de crianças em idade de crescimento. A terapia comportamental se faz extremamente necessária. Ela deve abordar não só a criança, mas também os responsáveis pela criança. Treinamentos envolvendo os pais com o intuito de entender e participar dessa mudança de comportamento são benéficos no curso do transtorno (FELDMAN HM, et al., 2014). 
Diante do exposto, o estudo visou discutir a importância do diagnóstico precoce do TDAH em crianças e suas formas de realização com o objetivo de garantir o tratamento adequado e, consequentemente, evitar danos ao desenvolvimento nesses períodos.

\section{REVISÃO BIBLIOGRÁFICA}

\section{Fisiopatologia}

O TDAH é caracterizado por uma alteração metabólica nas regiões pré-frontal e pré-motorado cérebro, resultado de desordem de funcionamento neurobiológico. Composto pela tríade: ausência de concentração, impulsividade e hiperatividade ou excesso de energia (SILVA ML, 2014).

Apesar de estudos de neuroimagem já terem identificado diversas variações estruturais e funcionais no cérebro associadas ao TDAH, a sua neurobiologia ainda não é totalmente compreendida, com muitos achados inconclusivos ou resultados contraditórios entre estudos. Os achados mais concretos dizem respeito à diminuição no volume total intracraniano e volumes subcorticais, especialmente os núcleos da base em indivíduos com TDAH (ALBAJARA S, et al., 2019)

A etiologia do TDAH é fortemente influenciada por fatores genéticos, sendo um dos transtornos psiquiátricos com maior herdabilidade estimada em 70- 80\% (FARAONE SV, 2018). Fatores exógenos também são influenciáveis, dividem-se em pré-natais, perinatais e natais. Os fatores pré-natais são compostos por infecções congênitas, intoxicações por medicamentosas ou substâncias venenosas, sangramentos, doenças maternas crônicas e traumatismo. Malformações pélvicas, prematuridade, anemia materna, sedação exagerada, hipotensão e hipertensão arterial, macrossomia fetal, malformações fetais, distúrbios respiratórios do recém-nascido como a doença da membrana hialina, síndrome de aspiração meconeal, incompatibilidade sanguínea materno-fetal, são exemplos de fatores perinatais. Tem grande influência também os fatores natais, como manobras de extração, distocias no parto, descolamento prematuro da placenta, anormalidades do cordão umbilical, parto cesáreo e por último os pós-natais, que são, traumatismo cranioencefálico, infecções neonatais, intoxicações e lesões expansivas (SOCIEDADE BRASILEIRA DE PEDIATRIA, 2018).

Meta-análises apontam associados ao TDAH os genes dos transportadores de serotonina 1B (HTR1B) e dopamina (D4 e D5), o gene codificador de uma proteína envolvida na liberação de neurotransmissores (SNAP25), o gene codificador da enzima catecol O-metiltransferase (COMT) e do brain- 31 derived neurotrophic factor (BDNF) e, exclusivamente em adultos, um gene envolvido em proliferação, sobrevivência e maturação neuronal (BAIAP2) (FARAONE SV, 2018).

O TDAH também foi relacionado à disfunção nas vias da serotonina (5-hidroxitriptamina [5-HT]), acetilcolina $(\mathrm{ACH})$, opioide e glutamato (GLU). As alterações nesses sistemas de neurotransmissores afetam a função das estruturas cerebrais que moderam a função executiva, a memória de trabalho, a regulação emocional e o processamento de recompensas (FARAONE SV, 2018).

\section{Comorbidades associadas}

O indivíduo que apresenta TDAH na infância, geralmente apresenta outras comorbidades associadas, devido a série de alterações psíquicas e sociais, causadas pela sua sintomatologia ou a comorbidade pode ser desenvolvida paralelamente. Além disso, o risco de desenvolvimento de depressão é por volta de 3 vezes maior em crianças com TDAH do que em crianças sem a doença. Portanto, fica evidente a importância do diagnóstico precoce para 0 monitoramento do desenvolvimento de outras doenças relacionadas (ASSOCIAÇÃO BRASILEIRA DO DÉFICIT DE ATENÇÃO, 2015).

Os danos causados pelo TDAH incluem o baixo desempenho escolar, e relacionamentos conflituosos, e comorbidades associadas como ansiedade e depressão. Sendo assim, existe uma propensão ao desenvolvimento de depressão na adolescência em indivíduos diagnosticados com TDAH na infância (POWELL V, et al., 2020). 
Em crianças com TDAH, cerca de metade apresentam pelo menos 1 transtorno de ansiedade. Sabe-se ainda que os transtornos de ansiedade estão associados ao baixo rendimento escolar, pois afeta a capacidade de memorizar as matérias passadas em sala de aula, prejudica a atenção e o interesse pelo assunto do que está sendo ensinado, porque a mente está focada no motivo da ansiedade, sendo assim, destaca-se que 47,9 indivíduos com TDAH e duas comorbidades de ansiedade concluíram o ensino médio, enquanto que 56,2 dos pacientes que tinha apenas TDAH concluíram o ensino médio, os primeiros perdiam mais dias letivos e tendiam a chegar atrasados à escola do que os segundos pacientes, assim nota-se a importância de diagnosticar precocemente essa comorbidade para melhorar a qualidade de vida e para que o rendimento escolar não seja prejudicado (SCIBERRAS E, et al., 2014 ).

O transtorno desafiador é caracterizado por comportamento desafiador e desobediente a figuras de autoridade, além de agressividade e teimosia. É mais comum entre crianças do sexo masculino. Indica-se que cerca de $28 \%$ das crianças que têm transtorno desafiador já tinham também o diagnóstico de TDAH, esse é um distúrbio externalizante, é essencial diferenciar os dois transtornos para manejar o tratamento do paciente e não tratar o TDO como sintoma da hiperatividade do paciente com TDAH (MOHAMMADI MR, et al., 2020).

Pode-se constatar que as comorbidades estão diretamente associadas ao TDAH, como por exemplo no caso de distúrbios internalizantes e externalizantes. O primeiro, destaca-se ansiedade e depressão indicando uma postura desatenta, relacionada ao déficit de atenção e os distúrbios externalizantes como comportamento opositor e distúrbio de conduta estão associados à hiperatividade, destacando uma postura mais agitada (OLASHORE AA, et al., 2020).

\section{Alterações sociais e cognitivas}

Embora possa ser invisível no contexto da vida cotidiana, o TDAH tem um sério impacto no bem-estar psicológico e social e no desempenho acadêmico dos indivíduos afetados. Pacientes podem apresentar sintomas de desatenção, hiperatividade ou impulsividade, desregulação emocional, prejuízo cognitivo e comorbidades. Podem ter prejuízo funcional com impacto negativo na qualidade de vida (MARTíNEZ MV, et al., 2017).

O prejuízo social no TDAH ocorre tanto dentro da família quanto com os pares. A relação entre pais e filhos com TDAH está associada a mais conflitos. Crianças com TDAH têm menos probabilidade de serem relatadas como tendo um bom relacionamento com seus irmãos. Mais da metade das crianças têm sérios problemas com relacionamentos com seus pares, e cerca de até $70 \%$, podem não ter amizades íntimas na infância. Crianças com TDAH são mais propensas a entrar em contato com grupos desviantes se a rejeição social continuar na adolescência. As dificuldades de socialização podem resultar em níveis mais elevados de ansiedade, que por sua vez, provavelmente intensificariam as respostas impulsivas e afetivamente não moduladas (BOOTH KVP, 2016; HADDAD AH, et al., 2021).

Apesar de ser um transtorno com rotineiro surgimento durante a infância, é comum que ele persista na idade adulta, causando prejuízos principalmente no desenvolvimento social, acadê-mico e profissional do paciente. O TDAH é mais frequente no sexo masculino, na proporção de 2:1 em crianças e de 1,6:1 nos adultos. Além disso, as mulheres frequentemente apresentam mais sintomas de desatenção quando comparado aos demais (LIMA CX e LIMA RF, 2018).

Indivíduos do sexo feminino, tidas como mais retraídas em vários aspectos da vida, caso se a hiperatividade fosse indicada como diagnostico comportamental, provavelmente seria mais fácil de se associar com algo que escapa das expectativas de modelos que prevalecem na sociedade. No entanto, a falta de atenção é a característica mais marcante nas meninas, fazendo com que haja uma fuga dos padrões impostos pela sociedade gerando então sentimento de ser inferior (BARBARINI TA, 2020).

Sendo assim o indivíduo detentor de TDAH não teria a capacidade de tomar decisões próprias e ter resolubilidade diante de problemas, devido às alterações neurofisiológicas. Essa mesma dificuldade de conseguir colocar a vida nos trilhos e conseguir ir adiante, é a mesma que causa dificuldade com relacionamentos e a falta de uma boa interação com a sociedade, associados a vários fracassos pessoais no 
decorrer da vida, fazendo com que haja maior probabilidade de outros riscos como envolvimento com crime, drogas, acidentes e até mesmo sintomas depressivos (MARTíNEZ MV, et al., 2017).

\section{Diagnóstico}

A fim de minimizar os danos agudos e a longo prazo causados pelo TDAH, é indispensável o diagnóstico precoce e o tratamento adequado do transtorno. O diagnóstico é essencialmente clínico e interdisciplinar. $O$ diagnóstico clínico se baseia nos critérios estabelecidos no DSM-5 da Associação Psiquiátrica Americana e na Classificação de Transtornos Mentais e de Comportamento (CID-10) da Organização Mundial da Saúde. É preconizado que a avaliação inclua a utilização de escalas e entrevistas com o paciente e seus familiares e professores, de acordo com a idade (LIMA CX e LIMA RF, 2018).

O médico da atenção primária deve se atentar para um possível caso de TDAH quando se tem um paciente na faixa etária entre 4 e 18 anos que apresente problemas acadêmicos ou comportamentais e sintomas de desatenção, hiperatividade ou impulsividade. Para fazer um diagnóstico em crianças, os médicos devem conduzir uma entrevista clínica com os pais, examinar e observar a criança e obter informações dos pais e professores por meio escalas de avaliação baseadas em TDAH (WOLRAICH ML, et al., 2019).

O DSM-5 define que os sintomas devem ter persistido por pelo menos 6 meses em um grau que é inconsistente com o nível de desenvolvimento e não deve ser melhor explicado por outro diagnóstico. Para atender aos critérios diagnósticos, crianças até 17 anos devem apresentar pelo menos 6 dos 9 sintomas em um ou ambos os domínios, devem estar presentes antes dos 12 anos de idade e devem também causar impacto negativo em 2 ou mais cenários (BOOTH KVP, 2016).

Conforme a manifestação dos sintomas, o TDAH pode variar na sua forma de apresentação. Pode ser classificado em combinado; predominantemente desatento; ou predominantemente hiperativo/impulsivo. A atual classificação do DSM-5 propõe ainda a classificação do transtorno como leve, moderado e grave, essa variação é de acordo com a quantidade de sintomas presentes e o grau de comprometimento que os mesmos causam na vida do paciente (LIMA CX e LIMA RF, 2018).

Para fazer um diagnóstico de TDAH, o médico deve determinar que os critérios do DSM-5 foram atendidos, incluindo a documentação de sintomas e comprometimento em mais de um ambiente principal (ou seja, social, acadêmico ou ocupacional), com informações obtidas principalmente de relatórios de pais ou responsáveis, professores, outros funcionários da escola e médicos de saúde mental que estão envolvidos no cuidado da criança ou do adolescente. Também deve-se descartar qualquer causa alternativa (WOLRAICH ML, et al., 2019).

\section{Tratamento}

O Transtorno do déficit de atenção com hiperatividade tem sua abrangência heterogênea, não apenas como um aspecto biológico, mas também com influência dos aspectos socioculturais, cognitivos, emocionais e comportamentais. Nesse sentido o tratamento se torna peculiar pois é necessária uma integração entre as estratégias terapêuticas farmacológicas e comportamentais (ARAGÃO L, et al., 2016). Existem evidências que mostram que as intervenções precoces proporcionadas pelo diagnóstico precoce de TDHA tem um melhor prognóstico para a criança com TDHA. Neste sentido, a terapia comportamental favorece o convívio social e o desenvolvimento da criança, porém o tratamento combinado com fármacos ainda é considerado a primeira escolha (TIMOTHY J, et al., 2018)

Contudo, antes de iniciar o tratamento farmacológico é importante compreender que a sinalização de norepinefrina e dopamina influenciam na otimização do desempenho do processo cognitivo de um paciente saudável. Porém estudos de neuroimagem do transportador de dopamina em humanos usando emissão de pósitrons (PET) sugerem que o transportador de dopamina tem maior atividade nos pacientes com TDAH do que em indivíduos saudáveis. Consequentemente a liberação da norepinefrina que é um produto do metabolismo da dopamina sofre alteração o que explica o transtorno pois em paciente saudáveis a neurotransmissão da norepinefrina regula importantes funções cognitivas como memória de trabalho e controle inibitório (ROHDE LA, et al. 2019; EDWIN FL, et al., 2018) 
Por conseguinte, o objetivo fundamental do tratamento medicamentoso é otimizar a neurotransmissão através dos glutamatérgica, corrigindo os níveis de dopamina e norepinefrina. Ensaios clínicos randomizados de curto prazo controlados por placebo envolvendo crianças com TDAH mostraram um benefício clinicamente significativo de medicamentos estimulantes derivados de metilfenidato e anfetaminas na redução da desatenção, hiperatividade e impulsividade (ROHDE LA, et al. 2019; EDWIN FL, et al., 2018)

O metilfenidato e as anfetaminas são responsáveis por inibir a dopamina e os transportadores de recaptação de noradrenalina. Os fármacos estimulantes que têm a liberação sustentada e de ação prolongada são geralmente preferíveis quando comparado aos agentes de ação curta porque permitem a administração de uma única dose matinal para melhorar os sintomas durante todo o dia escolar sem aumentar os efeitos adversos, sendo os mais comuns a perda de apetite e o retardo do sono (KRINZINGER H, et al., 2019)

Os medicamentos não estimulantes desempenham um papel importante no tratamento quando os estimulantes são contraindicados e têm muitos efeitos adversos. O mais utilizado é a atomoxetina que é um fármaco não estimulante inibidor do transporte de recaptação de norepinefrina. Entretanto em um estudo realizado com crianças tratadas com altas doses de atomoxetina ou metilfenidato devido ao tardio diagnóstico de TDAH e opção de intervenção apenas farmacológica resultaram no aumento significativo da frequência cardíaca e da pressão arterial (EDWIN FL, et al., 2018).

Portanto a terapia comportamental é essencial para o eficaz tratamento da doença pois pode influenciar no ambiente que as crianças vivem proporcionando progresso em inúmeras experiências motoras como escrever, jogar bola, correr, durante a intervenção. Além disso, corrobora para a diminuição da administração farmacológica nas crianças e consequentemente diminuindo os efeitos adversos de altas doses, como o aumento da frequência cardíaca. Os melhores resultados da terapia comportamental incluem ensinar aos pais como se comunicar sobre suas emoções, promovendo habilidades positivas de interação pai-filho e exigindo que os pais pratiquem a aplicação de técnicas de modificação de comportamento com seus filhos durante as sessões de treinamento além de proporcionar um treinamento intensivo de habilidades sociais durante um dia (OLIVEIRA CC, et al., 2018; KRINZINGER H, et al., 2019).

\section{CONSIDERAÇÕES FINAIS}

A partir da análise dos achados da literatura foi averiguado que é de suma importância o diagnóstico precoce de TDAH em crianças e adolescentes pois sua presença está diretamente associada a uma percepção prejudicada da qualidade de vida, do desempenho escolar, autoestima e desenvolvimento de transtornos psiquiátricos. Nesse sentido, verifica-se a necessidade de maior oferta e conhecimento de uma assistência especializada como neuropediatras e pediatras a fim de que ocorra um precoce diagnóstico da doença e um bom manejo do tratamento tanto farmacológico quanto comportamental. Além disso, é imprescindível incentivar as escolas a estarem atentas aos sinais e sintomas suspeitos buscando fazer um encaminhamento para um possível diagnóstico precoce.

\section{REFERÊNCIAS}

1. ALBAJARA S, et al. Neuroimagem estrutural e funcional no transtorno de déficit de atenção / hiperatividade. Dev Med Child Neurol, 61: 399-405, 2019

2. ARAGÃO L, et al. Diagnosis and treatment of ADHD: Criteria used by different professional categories. Liberabit, 2016; 22(1): 111-120

3. ASSOCIAÇÃO BRASILEIRA DO DÉFICIT DE ATENÇÃO. Quadro Clínico. Brasil, 2015. Disponível em: https://tdah.org.br/quadro-clinico/. Acesso em: 15 junho 2021

4. BANASCHEWSKI T, et al. Attention-Deficit/Hyperactivity Disorder. Deutsches Arzteblatt international, 2017;114(9): 149-159.

5. BARBARINI TA. Corpos, "Mentes", emoções: uma análise sobre TDAH e socialização infantil. Revista Psicologia \& Sociedade, 2020.

6. BOOTH KVP. Attention-Deficit/Hyperactivity Disorder (ADHD) in Children Born Preterm and With Poor Fetal Growth. Rev. NeoReviews, 2016. 
7. CARVALHO DMS, et al. Transtorno de Déficit de Atenção com Hiperatividade (TDAH) em crianças e adolescentes atendidos em um ambulatório universitário: descrição e análise. In: Anais do encontro do programa de pós graduação em ciências farmacêuticas, 2017.

8. DIAGNOSTIC AND STATISTICAL MANUAL OF MENTAL DISORDERS (DSM-5). Diagnostic and Statistical Manual of Mental Disorders. American Psychiatric Association, 2013; 59-66p.

9. EDWIN FL, et al. O efeito do metilfenidato e da atomoxetina na frequência cardíaca e na pressão arterial sistólica em jovens e adultos com transtorno de déficit de atenção e hiperatividade (TDAH): revisão sistemática, meta-análise e meta-regressão. International Journal of Environmental Research and public Health, 2018.

10. EFFGEM V, et al. A visão de profissionais de saúde acerca do TDAH-processo diagnóstico e práticas de tratamento. Construção psicopedagógica, 2017; 25(26): 34-45.

11. FARAONE SV. The pharmacology of amphetamine and methylphenidate: Relevance to the neurobiology of attention deficit/hyperactivity disorder and other psychiatric comorbidities. Neurosci Biobehav Rev. 2018; 87: 255-70

12. FELDMAN HM, et al. Attention deficit-Hyperactivity Disorder in Children and adolescents. The New England Journal of Medicine, $2014 ; 370: 838-846$

13. HADDAD AH, et al. Comprometimento da Personalidade em Crianças e Adolescentes com TDAH. Rev. Paidéia, 2021, 31(3105): 1-9.

12. KRINZINGER $\mathrm{H}$, et al. Neurological and psychiatric adverse effects of long-term methylphenidate treatment in ADHD: A map of the current evidence, 2019; 107: 945-968

13. LIMA CX, LIMA RF. Consequências do transtorno do déficit de atenção e hiperatividade (TDAH) na idade adulta. Rev. Psicopedagogia, 2018; 35(106): 61-72.

14. MARTíNEZ MV, et al. Quality of life in children with attention deficit hyperactivity disorder (ADHD). Rev Pediatr Aten Primaria, 2017; 19:31-9.

15. MOHAMMADI MR, et al. Lifetime prevalence, sociodemographic predictors, and comorbidities of oppositional defiant disorder: the National Epidemiology of Iranian Child and Adolescent Psychiatric disorders (IRCAP). REV. Brasileira de psiquiatria, 2020; 162-167p.

16. OLASHORE AA, et al. Transtorno de déficit de atenção e hiperatividade em crianças em idade escolar em Gaborone, Botswana: Comorbidade e fatores de risco. South african jornal of psychiatry, 2020; 26: 1525.

17. OLIVEIRA CC, et al. Motor characteristics of students with attention Deficit Hyperactivity Disorder,2018; 26(3): 590$600 p$

18. POWELL V, et al. $\mathrm{O}$ que explica a ligação entre o TDAH na infância e a depressão na adolescência? Investigar o papel das relações entre pares e realização acadêmica. Eur Child Adolesc Psychiatry, 2020; 29: 1581-1591.

19. SCIBERRAS E, et al. Anxiety in children with attention déficit hyperactivity disorder. Official Journal of the american academy of Pediatrics, 2014; 133(5): 801-8.

20. SIGNOR RCF, SANTANA APO. A constituição da subjetividade na criança com diagnóstico de Transtorno de Déficit de Atenção e Hiperatividade. Bakhtiniana, Rev. Estud. Discurso, 2020; 15(2): 210-228,

21. SILVA ML. Mentes inquietas: TDAH; desatenção, hiperatividade e impulsividade. (4a ed.), Globo. 304p, 2014

22. SOCIEDADE BRASILEIRA DE PEDIATRIA. In: Departamento Científico Pediatria do Desenvolvimento e Comportamento SBP, 2018. Disponível: https://www.sbp.com.br/departamentos-cientificos. Acessado em: 09 de agosto de 2021.

23. TIMOTHY J, et al. Transtorno de déficit de atenção-hiperatividade e mês de matrícula escolar. The new england Journal of Medicine, 2018; 379: 2122-2130

24. WOLRAICH ML, et al. Clinical Practice Guideline for the Diagnosis, Evaluation, and Treatment of Attention Deficit Hyperactivity Disorder in Children and Adolescents. Official Journal of the american Academy of Pediatrics, 2019; 144(4): 1-19p 